\title{
A Brief Overview of AFM Force Distance Spectroscopy
}

\author{
Devendra Khatiwada and Shobha Kanta Lamichhane \\ Department of Physics, PN Campus, Pokhara, Tribhuvan University, Nepal \\ prit_khatiwada@yahoo.com
}

\section{Abstract}

Study of forces between the sample and the AFM cantilever tip is the basic in AFM force distance study. AFM plays vital role and acts as the Hooken's response giving rise to the force measurement. Force characterization depends on various conditions. That yield basic theories like JKR , DMT interaction etc. have been using for its explanation.

Key words:- AFM, Hooken's response, UHV, JKR, DMT

\section{Introduction}

Force distance curve plays a vital role for the explanation of different types of interaction and the underlying physics for the interaction. The force is not same as in AFM is brought in or out (attraction and retraction). Force distance curve is the study of interaction force between the cluster of atoms in the AFM cantilever tip and the sample surface. What we measure in AFM is the distance between the cantilever and the sample $(\mathrm{Z})$, not the distance between the sample and the tip (d). The distance controlled during the measurement is not the actual tip-sample distance (d), but the distance $(\mathrm{Z})$ between sample surface and the rest position of the cantilever. These two distances differ because of cantilever deflection $\delta_{c}$ and of the sample deformation $\delta_{\mathrm{s}}[1]$. These quantities are related as follows:

$$
\mathrm{D}=\mathrm{Z}-\left(\delta_{\mathrm{c}}+\delta_{\mathrm{s}}\right)
$$

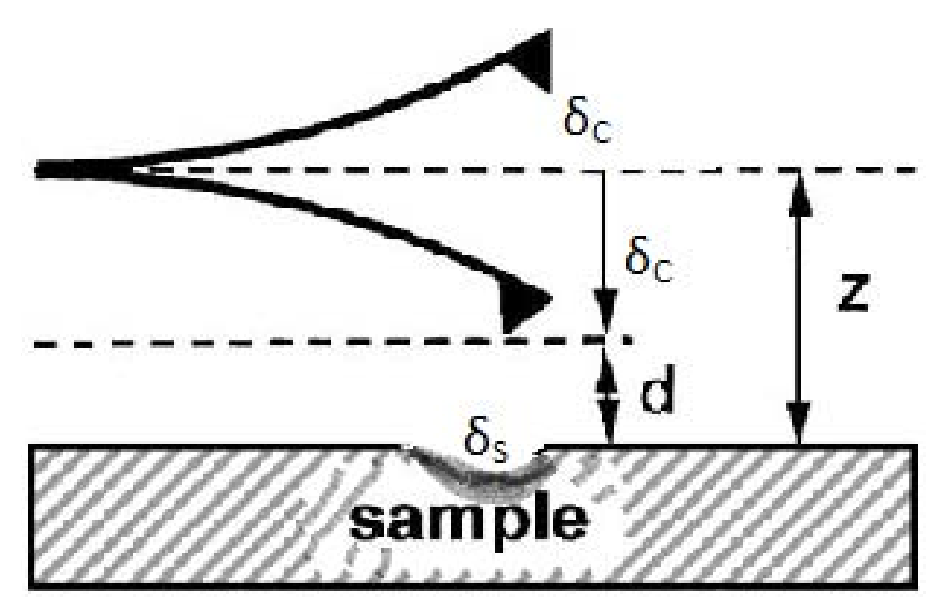

Fig.1: Cantilever deflection.

Where, $d$ is the smallest gap between the tip and the sample. So the obtained curve is the $\mathrm{F}$ vs. $\mathrm{Z}$ curve rather than $\mathrm{F}$ vs. $\mathrm{d}$ curve. However the two can be converted from one to the other. Lots of interesting Physics lies in Force distance curve. Force is effective among the cluster of atoms in tip and the sample. This can be simply explained by the Lenard zone potential curve, which is the nature of the obtained curve due to the interaction.

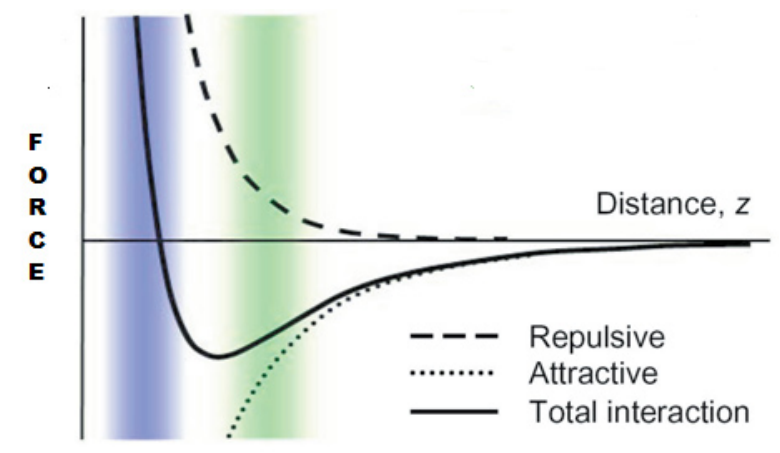

Fig 2: - Lennard-Jones potential curve.

As we see to the right of the curve in the attractive regime, force is negative which gives explanation of the inverse square law of forces. In case of AFM this is the region where the AFM cantilever tip is brought from some extent where $d$ is equal to zero (chosen arbitrary) and reaches to the attractive regime due the force of adhesion. At certain distance to the sample surface, tip of the cantilever 'snaps in'. It is the case when the adhesion force is much larger than the force due to cantilever. The reverse phenomenon occurs during the retraction called 'snaps out' as we pull the tip away. This region explains about the well known Haymaker constant, Vander Waals force etc and interesting part of physics lies here. The force of adhesion may be due to the Vander walls 
force (caused due to polarization) or capillary force (due to ambient condition) and others. The magnitude of these forces depends on the true contact area and nature of the attractive forces holding the surface together [2].The basic analysis of force measurement can be done from different applied theories like JKR, DMT, Maugis [3], Carpick, Ogletree and Salmeron (COS) etc [4]. Whereas, if we go to the left side of the curve, we get the repulsive regime which explain about the elasticity and inelasticity of the sample for AFM measurement.

Force distance curve is some time hysteretic. Where, the hysteresis occurs due to necking of the soft sample itself as can be seen in the JKR interaction model. Thus JKR interaction is called the dissipative interaction where there is net loss of energy. Whereas DMT type of interaction are called the conservative interaction as the force depends on the gap between the tip and sample.

\section{Force measuring technique}

The force due to cantilever (about $100-200 \mu \mathrm{m}$ ) is simply the force due to its Hooken's response given by Hook's law as:-

$$
\mathrm{F}=\mathrm{k} \delta
$$

Where, $\mathrm{k}$ is the spring constant of the cantilever and $\delta$ is the deflection of the cantilever. We use the given value of spring constant and the deflection can be calculated from optical beam deflection method through PSPD (Position sensitive photo detector). For the non given value of $\mathrm{k}$ we obtain it by beam geometry where $\mathrm{k}$ is given as:-

$$
\mathrm{k}=3 \mathrm{EI} / \mathrm{L}^{3}
$$

Where,

E is Young's modulus of elasticity

I is the moment of inertia which for the rectangular beam is $\mathrm{I}=\mathrm{bd}^{3} / 12, \mathrm{~b}$ is the breadth and $\mathrm{d}$ is the thickness of cantilever.

$\mathrm{L}$ is the length of the cantilever beam.

Several other methods have been illustrated by John Ralston [5]. This cantilever force balances the force due to adhesion as a result of which the force of adhesion can be calibrated. The pull of force for the JKR type of interaction is given as:-

$$
\mathrm{F}_{\text {pull off }}=(-3 / 2) \pi \Upsilon R
$$

Where,

$\Upsilon$ is the net force of adhesion of sample and the tip, $\mathrm{R}$ is the effective radius between the tip and the sample.

This theory is given for the compliant material having large sphere radii with short range and strong adhesion force. For the stiff material having small sphere radii with weak and long range adhesion force DMT theory is used according to which the force of adhesion is given as [6].

$$
F_{\text {pull -off }}=-2 \pi \Upsilon R
$$

Where, the indices are as explained above. The pull off force is the force to overcome the adhesion. The adhesion force could include Vander Waals force, capillary force or the electrostatic force. An excellent text explaining intermolecular and surface forces is given by Israelachvili [7].We basically ignore all the ambient condition and perform the set up in zero relative humidity condition.

\section{Result and Discussion}

AFM with a given value of spring constant was taken and was used for the force analysis. It is to be noted that the spring constant of the cantilever must be less than that of the equivalent spring constant between atoms in the solid to avoid the possibility of the spring actually pushes atoms aside [7] . Experimentally obtained Force distance curve using silicon tip cantilever for iron substrate is explained in figure (i).

The graph is like the Lenard zone potential as discussed above. The curve for attraction and retraction is shown by two different lines and the position of cantilever during the process is illustrated in the ideal graph (b). Position (i) shows the cantilever when it is at far sight and no adhesion force acts. Position (ii) is where cantilever exerts force of adhesion and suddenly 'snaps in' during which the cantilever moves inward. The repulsive interaction thus comes into play which balances the cantilever to its position (iii). Further inward movement will result in repulsive force which bends the cantilever with same $\mathrm{z}$ (iv). Again as the cantilever is retraced (moved back) the reverse phenomenon occurs, called retraction shown by the position (v), (vi) and (vii). The AFM set up is also shown in fig (a). 


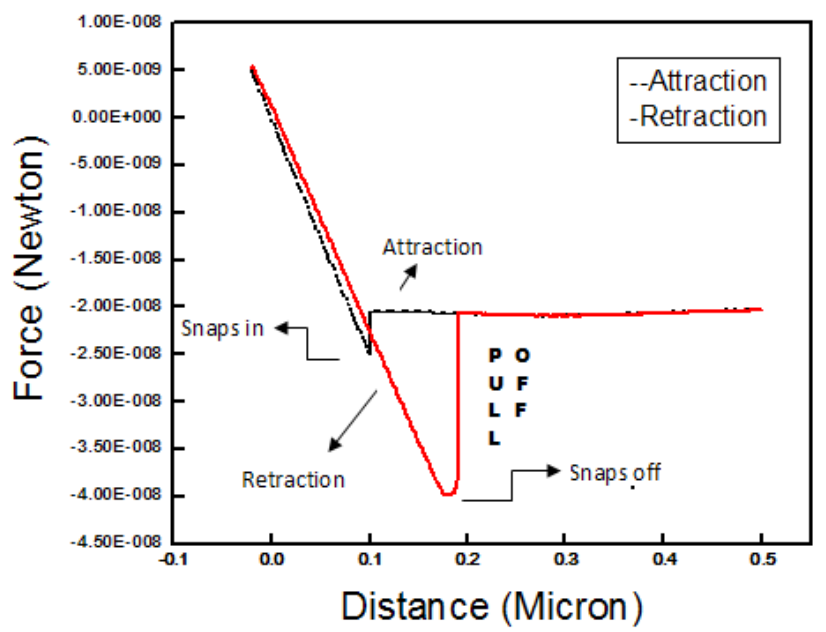

Fig (i):-Force distance curve for iron substrate using silicon tip in $A F M$.

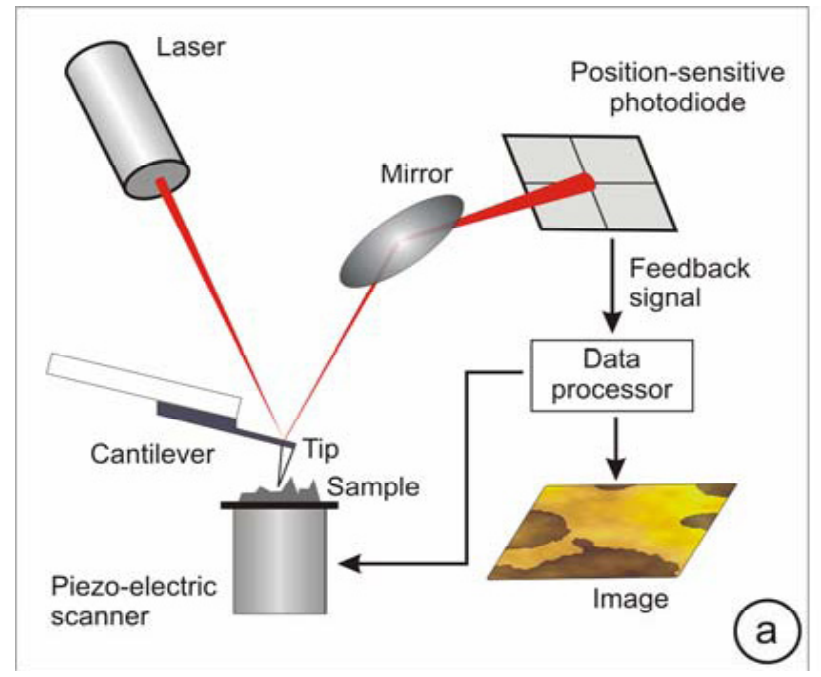

Fig: - a) AFM set up for force calibration.

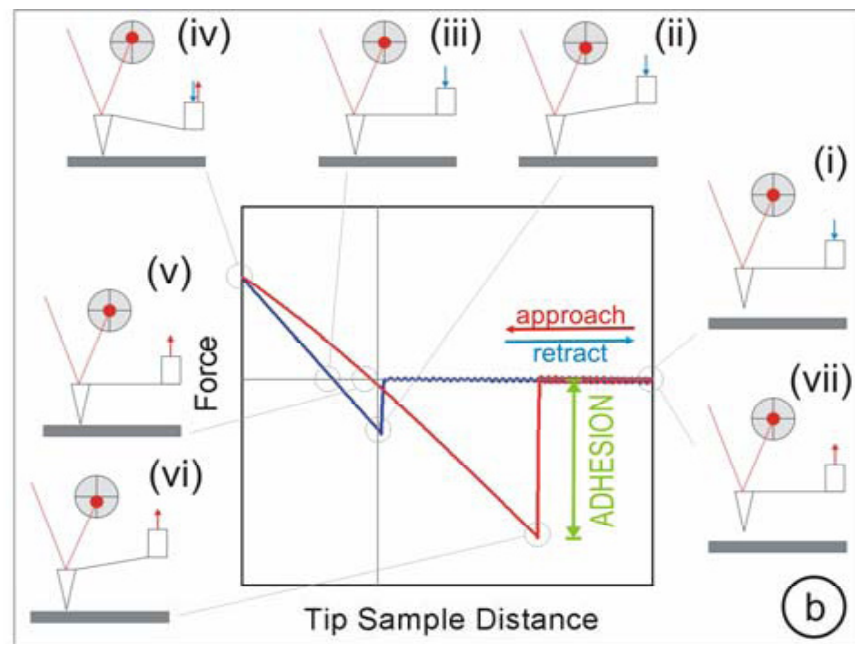

b) Ideal force distance curve showing attraction and retraction.
According to DMT mechanism the adhesive force is given as

$$
\Upsilon=\left(-\mathrm{F}_{\text {pull-off }} / 2 \pi R\right)
$$

And according to JKR mechanism the value of adhesion is given as

$$
\Upsilon=\left(-2 \mathrm{~F}_{\text {pull-off }} / 3 \pi \mathrm{R}\right)
$$

The value of $\mathrm{R}$ is given as:-

$$
1 / \mathrm{R}=1 / \mathrm{R}_{1}+1 / \mathrm{R}_{2}
$$

Where, $R_{1}$ and $R_{2}$ are radius of the tip and the sample respectively. The sample is taken to be too stiff, so the value of $R_{2}$ is taken to be infinite. Hence,

$\mathrm{R}=\mathrm{R}_{1}=$ Radius of the silicon tip of AFM which was taken to be $5 \mathrm{~nm}$ (AFM catalogue $\mathrm{R}$ is $5-10 \mathrm{~nm}$, we take $5 \mathrm{~nm}$ )

For the value of particular pull off force from the graph we calculate the value of adhesion using DMT theories considering the sample to be stiff. For the pull off force $-3.00 \mathrm{E}-008$ we get the value for the force of adhesion as

For DMT type of interaction we get,

$$
\begin{aligned}
\Upsilon & =(3.00 \mathrm{E}-008) / 2 \times(3.14) \times\left(5 \times 10^{-9}\right) \\
& =0.95 \times 10^{-6} \mathrm{~N} / \mathrm{m}
\end{aligned}
$$

Thus, value of force of adhesion is found $0.95 \times 10^{-6}$. If the process was carried considering the compliant sample where no long interaction acts we would better use the JKR type of interaction. In case of UHV (ultra high vacuum) the two theories would be applicable under the consideration of the nature of substrate and the tip. Several recent studies has investigated that the adhesion force between AFM tip and sample depends strongly on whether the substance is hydrophobic or hydrophilic. If we consider the instantaneous polarization of atoms interacting with the surrounding atoms we take in account of the Vander Waals forces for the adhesion given as

$$
\mathrm{V}_{\mathrm{A}}=\mathrm{HR} / 6 \mathrm{z}^{2}
$$

Where, $\mathrm{H}$ is called the Hamaker constant, $\mathrm{R}$ is the radius of tip and $\mathrm{z}$ is the distance between the cantilever and the substrate. Hence the well known Hamaker constant can be determined from the consideration of the Vander Waals force to be only the force of adhesion. However the value of Hamaker constant as calculated by Lifshitz expression is important [9]. In case of ambient condition 
we do consider the capillary force as the part of adhesion. So for the flat surface joined by the liquid bridge the force due to adhesion given by the Laplace pressure is given by

$$
\mathrm{F}=4 \pi \mathrm{R} \Upsilon_{\mathrm{LV}} \cos \theta
$$

\section{References}

[1] B. Cappella, G. Dietler, 1999. Force-distance curves by atomic force microscopy Dietler/Surface Science Reports 34.

Where $\Upsilon_{L V}$ is the surface tension of the liquid in the condensate

[2] M.Rosoff(ed), 2002. Nano surface Chemistry, Marcel Dekker Inc NY, USA.

and $\theta$ is the contact angle of this liquid on the solid.

[3] D. Maugis, 1992. J. Colloid Interf. Sci. 150, 243-269.

AFM measurements performed under ambient condition are thus influenced by the presence of vapors, in particular by water vapor [10]. This capillary force can also be illustrated as

$$
\mathrm{F}_{\text {capillary }}=\Upsilon \mathrm{A} / \mathrm{r}_{\mathrm{k}}=\Upsilon 2 \pi \mathrm{Rd} / \mathrm{r}_{\mathrm{k}}
$$

[5] John Ralston, 2005. Pure and applied chemistry vol 77 no12, pp 2149-2170.

[6] D. S. Grierson, E. E. Flater and R. W. Carpick; 2005. Where, $\Upsilon$ is the net surface tension, $A$ is contact area of the meniscus given as $A=2 \pi R d, R$ is tip radius and $d$ is tip radius depth, $\mathbf{r}_{\mathbf{k}}$ the Kelvin radius.

\section{Conclusion}

Force distance curve has thus provided us a bridge of knowledge for the study of different interaction and its measurement. The region of snaps in and snaps out are still the points to be analyzed for the AFM researcher where the huge Physics has still been hidden. The hysteretic effect opens the path to study and understand the laws of Physics.

Different consideration has to be taken for the force analysis under different theories and the most reliable. Even we discussed the well applied theory the violation may occur in whatever we have, under some extent as the violation of JKR was found due to small size of contact radius, compared to the thickness leading to DMT like interaction. Much research has been undertaken for appropriate theory under different sample like the biological and the study of Nano Tribology. We believe to the famous saying by Feynman in his lecture that 'There's Plenty of Room at the Bottom' and search for new events in Nano science.

\section{Acknowledgement}

I am grateful to Dr. Shobha Kanta Lamichhane and my entire colleague for their kind co operation has led a step for this. I would like to honor PN campus for providing me the platform.

Accounting for the JKR-DMT transition in adhesion and friction measurements with atomic force microscopy $J$. Adhesion Sci. Technol., Vol. 19, No. 3-5, pp. 291-311.

JN. Israelachvili and D. Tabor, 1972. intermolecular and surface force Proc. R. Soc. Lond. A 331, 19-38.

S.K. Lamichhane, 2005. PhD thesis on silicon substrate, JNU university New Delhi -110067.

[9] J.C Froberg et al 1999. /int j Miner process 56.

[10] E.Meyer, Atomic Force Microscope Progress in Surface Science, Vol. 41, pp. 3-49. 